\title{
Numerical-renormalization-group approach to Anderson localization
}

\author{
P. M. Bell and A. MacKinnon \\ Condensed Matter Theory Group, The Blackett Laboratory, Imperial College, Prince Consort Road, \\ London SW72BZ, United Kingdom
}

(Received 1 September 1994)

\begin{abstract}
We have studied the behavior of the conductance in large disordered systems using a numerical real-space renormalization-group ( $R G$ ) technique. Using a distribution of scattering matrices as the fundamental building blocks of our system, very large structures can be studied by combining the blocks in the appropriate way and performing a RG step at each stage. The method is sufficiently general that it allows several RG techniques to be used and thus be compared with one another. We examine the conductance behavior in two and three dimensions with increasing system size for different shaped building blocks and different initial disorders. We compare the results with other work. Our results are extremely sensitive to the detailed implementation of each RG method used and therefore we suggest that all localization results obtained via RG methods must be interpreted with caution.
\end{abstract}

\section{INTRODUCTION}

Although the physics of Anderson localization ${ }^{1,2}$ is well understood in one dimension (1D) ${ }^{3-6}$ there are still unresolved questions concerning the behaviour of systems near the metal-insulator transition in $3 \mathrm{D}$. In particular, there is disagreement about the values of the critical exponent (to the first decimal place) $)^{7-9}$ and the behavior of the conductance is still not well understood..$^{10-13}$

The most widely used numerical method for studying localization is the finite size scaling technique. ${ }^{14,15} \mathrm{Al}$ though it has been quite successful in describing various aspects of localization, it requires vast amounts of CPU time and makes the study of very large systems almost impossible. An alternative method is the vector recursion technique. ${ }^{16}$ It produces conflicting results (compared to the finite size scaling method) about the nature of the localization, but it too suffers from the fact that large systems are inaccessible.

An alternative technique which can be used to study Anderson localization is the real-space renormalizationgroup (RG) approach, developed by Wilson. ${ }^{17}$ It allows much larger systems to be studied in a fraction of the time taken by finite size scaling methods. However, one has to be extremely careful when applying this technique to the localization problem. The first attempt was made by Lee ${ }^{18}$ from which he concluded that there existed a metal-insulator transition in $2 \mathrm{D}$, in contrast to the scaling theory. ${ }^{2}$ This was later shown to be incorrect. ${ }^{19}$

Another attempt was made by Shapiro and co-workers using the scattering properties of a lattice rather than the more conventional Hamiltonian. ${ }^{11,20}$ However, there are some shortcomings in their approach: They do not include the dimensionality of the lattice in a proper way and they severely overestimate the conductance fluctuations in the weak scattering and metallic regime.

Recently, two more real-space renormalization-group methods have been reapplied to the localization problem.
White and Noack ${ }^{21,22}$ successfully described the onedimensional case and explained why previous attempts, based on the tight-binding Hamiltonian method, gave wrong answers. However, they are still some way from being able to describe Anderson localization in 2D and 3D. Finally, Dasgupta et $a$. $^{23}$ studied two-dimensional localization, using the vector recursion technique as a means to replace their large tight-binding lattice by a single site.

In this paper, we show how to improve on the approach of Shapiro and co-workers and by trying several different RG techniques we show that some caution must be used when examining the claims of Dasgupta et al.

\section{THEORY}

Consider some fundamental building blocks of a large system. In $2 \mathrm{D}$, this object could be a triangle or a square; see Fig. 1. If the object causes scattering to take place, then we can represent it mathematically by its scattering matrix. For the triangular scatterer in Fig. 1,

$$
\left(\begin{array}{c}
B_{1} \\
B_{2} \\
B_{3}
\end{array}\right)=\left(\begin{array}{ccc}
{ }^{1} r_{1} & { }^{1} t_{12} & { }^{1} t_{13} \\
{ }^{1} t_{21} & { }^{1} r_{2} & { }^{1} t_{23} \\
{ }^{1} t_{31} & { }^{1} t_{32} & { }^{1} r_{3}
\end{array}\right)\left(\begin{array}{c}
A_{1} \\
A_{2} \\
A_{3}
\end{array}\right),
$$

where we use the following notation: ${ }^{n} r_{i}$ is the complex reflection coefficient from the $i$ th face of the $n$th scatterer and ${ }^{n} t_{i j}$ is the complex transmission coefficient from face $i$ to face $j$ of the $n$th scatterer. $A_{i}$ and $B_{i}$ refer to the input and output wave amplitudes respectively. For other scatterers the notation is the same, the only difference being the size of the scattering matrix required to represent the object.

By joining the input and output channels of one scattering object to another, it is possible to create a larger scattering object; see Fig. 2. Mathematically, we must 

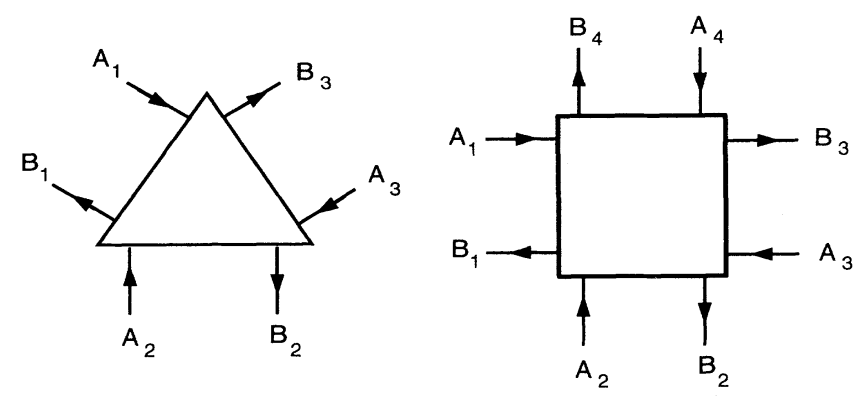

FIG. 1. Two simple scattering objects in 2D. The leads in and out of the scattering blocks are channels through which waves can scatter into and out of the object.

represent this by a new scattering matrix. The scattering matrix of the first object is given by

$$
\left(\begin{array}{c}
B_{1} \\
B_{2} \\
C_{2} \\
B_{6}
\end{array}\right)=\left(\begin{array}{cccc}
{ }^{1} r_{1} & { }^{1} t_{12} & { }^{1} t_{13} & { }^{1} t_{14} \\
{ }^{1} t_{12} & { }^{1} r_{2} & { }^{1} t_{23} & { }^{1} t_{24} \\
{ }^{1} t_{13} & { }^{1} t_{23} & { }^{1} r_{3} & { }^{1} t_{34} \\
{ }^{1} t_{14} & { }^{1} t_{24} & { }^{1} t_{34} & { }^{1} r_{4}
\end{array}\right)\left(\begin{array}{c}
A_{1} \\
A_{2} \\
C_{1} \\
A_{6}
\end{array}\right)
$$

and the second object has

$$
\left(\begin{array}{c}
C_{1} \\
B_{3} \\
B_{4} \\
B_{5}
\end{array}\right)=\left(\begin{array}{cccc}
{ }^{2} r_{1} & { }^{2} t_{12} & { }^{2} t_{13} & { }^{2} t_{14} \\
{ }^{2} t_{12} & { }^{2} r_{2} & { }^{2} t_{23} & { }^{2} t_{24} \\
{ }^{2} t_{13} & { }^{2} t_{23} & { }^{2} r_{3} & { }^{2} t_{34} \\
{ }^{2} t_{14} & { }^{2} t_{24} & { }^{2} t_{34} & { }^{2} r_{4}
\end{array}\right)\left(\begin{array}{c}
C_{2} \\
A_{3} \\
A_{4} \\
A_{5}
\end{array}\right)
$$

We now write these two equations as a single matrix equation having the form

$$
\left(\begin{array}{c}
\mathbf{B} \\
\mathbf{C}
\end{array}\right)=\left(\begin{array}{ll}
\tilde{S}_{11} & \tilde{S}_{12} \\
\tilde{S}_{21} & \tilde{S}_{22}
\end{array}\right)\left(\begin{array}{c}
\mathbf{A} \\
\mathbf{C}
\end{array}\right)
$$

where $\mathbf{A}=\left(A_{1}, \ldots, A_{6}\right), \mathbf{B}=\left(B_{1}, \ldots, B_{6}\right)$, and $\mathbf{C}=$ $\left(C_{1}, C_{2}\right)$. The submatrices of the scattering matrix $\tilde{S}_{11}, \tilde{S}_{12}, \tilde{S}_{21}$, and $\tilde{S}_{22}$, contain the relevant matrix elements of Eqs. (2) and (3) and where an output amplitude is not directly related to an input amplitude (e.g., $B_{1}$ and $A_{3}$ ), a zero is placed in the $8 \times 8$ matrix. Equation (4) can be written explicitly as

$$
\begin{aligned}
& \mathbf{B}=\tilde{S}_{11} \mathbf{A}+\tilde{S}_{12} \mathbf{C}, \\
& \mathbf{C}=\tilde{S}_{21} \mathbf{A}+\tilde{S}_{22} \mathbf{C} .
\end{aligned}
$$

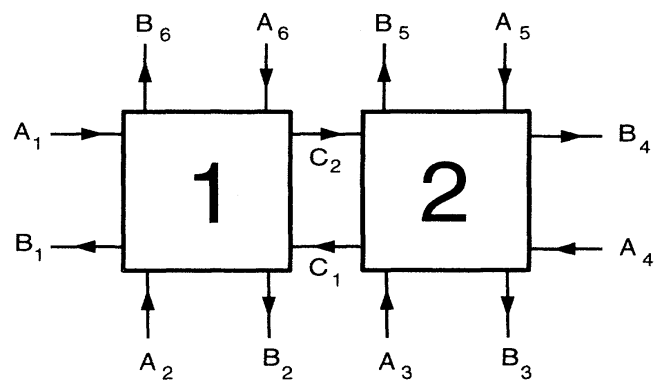

FIG. 2. By joining the input and output amplitudes of two scatterers together it is possible to create a larger system.
Rearranging Eq. (6) gives an expression for $\mathbf{C}$ which can be substituted into Eq. (5) to eliminate the internal amplitudes $\mathbf{C}$, and give an expression for the output amplitudes in terms of the input amplitudes only. Thus the scattering matrix $\tilde{S}$ of this larger object can be found and is given by

$$
\tilde{S}=\tilde{S}_{11}+\tilde{S}_{12}\left(1-\tilde{S}_{22}\right)^{-1} \tilde{S}_{21},
$$

where the multiple scattering is contained within the expression $\left(1-\tilde{S}_{22}\right)^{-1}$. This can be used continuously to build larger and larger systems and is not restricted to any particular shape of scatterer or dimension. Of course, for more complicated scatterers the size of the matrices becomes larger, making Eq. (7) more laborious to evaluate.

This process could be repeated indefinitely to create a large system, for which the scattering matrix would be known. However, the matrix will quickly become too large to manipulate or store in a computer and hence is of little practical use in calculating the transport properties of the infinite lattice. For this reason we make use of the numerical real-space renormalization group: If we join together four triangular or square scatterers, we end up with an object which resembles the original scatterer; see Fig. 3. The purpose of the RG approach is to replace this scattering object by a smaller one which nonetheless contains all the relevant information of the large object. Thus the approach we adopt is to try to replace the scattering matrix of the large object by a matrix with the same dimensions as the original scattering matrices. To do this we must reduce the number of channels on each face of the large object or decrease the space of the large object's scattering matrix. We can do this in a number of different ways.

\section{A. RG approach 1: Current conservation}

Suppose we reduce the dimensions of the scattering matrix, but ensure that the new matrix describes the same current flowing between faces of the scatterer. This would seem a natural thing to do since, ultimately, it is the conductance which we are most interested in and it is also a natural generalization of the idea of the conductance as the only relevant scaling variable. ${ }^{2}$ Consider Fig. $3(\mathrm{a})$. Before applying the RG procedure the scattering matrix of this large triangular scatterer can be written as

$$
\tilde{S}=\left(\begin{array}{cccccc}
{ }^{11} r_{1} & { }^{12} r_{1} & { }^{11} t_{12} & { }^{12} t_{12} & { }^{11} t_{13} & { }^{12} t_{13} \\
\vdots & { }^{22} r_{1} & { }^{21} t_{12} & { }^{22} t_{12} & { }^{21} t_{13} & { }^{22} t_{13} \\
\vdots & \ddots & { }^{11} r_{2} & { }^{12} r_{2} & { }^{11} t_{23} & { }^{12} t_{23} \\
\vdots & & \ddots & { }^{22} r_{2} & { }^{21} t_{23} & { }^{22} t_{23} \\
\vdots & & & \ddots & { }^{11} r_{3} & { }^{12} r_{3} \\
\cdots & \ldots & \cdots & \cdots & \cdots & { }^{22} r_{3}
\end{array}\right),
$$

where the notation has changed slightly: The superscripts denote the channels on each face through which 
transmission or reflection occurs. The matrix is symmetric if we have no external magnetic field. If we feed a wave of unit amplitude into face 1 , then the dimensionless conductance measured on face 2 will be (from the Landauer formula ${ }^{24}$ )

$$
g_{12}=\frac{1}{2}\left[\left|{ }^{11} t_{12}\right|^{2}+\left|{ }^{12} t_{12}\right|^{2}+\left|{ }^{21} t_{12}\right|^{2}+\left|{ }^{22} t_{12}\right|^{2}\right] .
$$

Similarly, the conductance measured on face 1 due to reflection is

$$
g_{1}=\frac{1}{2}\left[\left|{ }^{11} r_{1}\right|^{2}+\left.\left.\right|^{22} r_{1}\right|^{2}+\left.\left.2\right|^{12} r_{1}\right|^{2}\right]
$$

and the process can be repeated for all reflections and transmissions. Now we replace each conductance with a single reflection or transmission amplitude. These will be the amplitudes of matrix elements of the renormalized scattering matrix $\tilde{S}^{\mathrm{RG}}$,

$$
\left.\right|^{\mathrm{RG}} r_{i} \mid=\sqrt{g_{i}}, \quad i=1,2,3,
$$
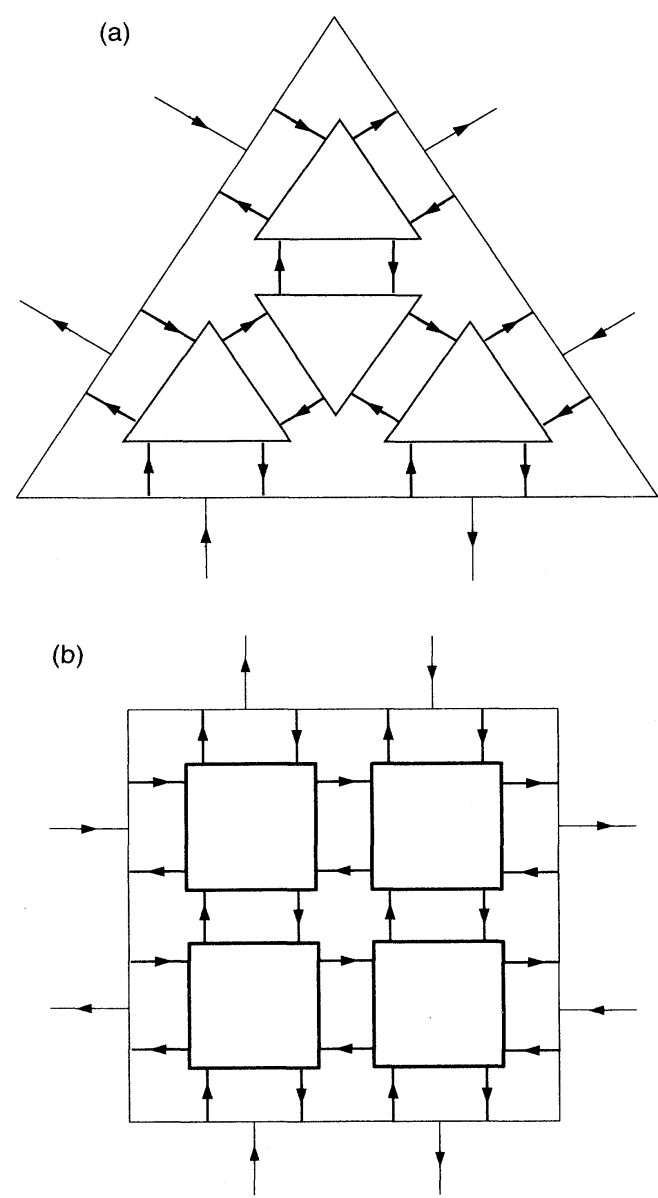

FIG. 3. In both cases a large scattering object, which resembles the original scattering objects, has been created by joining the input and output channels of four scatterers. The purpose of the RG technique is to reduce the number of channels on each face of the large object without losing relevant information.

$$
\left.\right|^{\mathrm{RG}} t_{i j} \mid=\sqrt{g_{i j}}, \quad i \neq j .
$$

The price which has been paid in using this RG procedure is the loss of the phase information. Fortunately, the unitarity condition provides the way to recover the phases. The condition of unitarity is given by

$$
\tilde{S}^{\mathrm{RG}} \tilde{S}^{\mathrm{RG} \dagger}=\mathbf{1}
$$

Suppose the reflection phase from each face of the scatterer is known. In fact, we assume it is a random variable, which is valid if we have a disordered system. ${ }^{25}$ Then, perform the following transformation to remove the phases from the diagonal elements of $\tilde{S}^{\mathrm{RG}}$ :

$$
\tilde{S}^{\prime}=\tilde{\Lambda} \tilde{S}^{\mathrm{RG}} \tilde{\Lambda}
$$

where

$$
\tilde{\Lambda}=\operatorname{diag}\left[\exp \left(-\mathrm{i} \theta_{1} / 2\right), \exp \left(-\mathrm{i} \theta_{2} / 2\right), \exp \left(-\mathrm{i} \theta_{3} / 2\right)\right]
$$

and $\theta_{1}, \theta_{2}$, and $\theta_{3}$ are the reflection phases from faces 1 , 2 , and 3 , respectively. The unitarity condition now gives us three diagonal equations and six off-diagonal equations, only three of which are independent. The three diagonal equations give the conditions of current conservation and provide a useful test that the $R G$ procedure has been successful up to this point. The three independent off-diagonal equations can be solved analytically and thus the phase information is obtained. If, however, we wish to study more complicated scatterers such as squares or cubes then we must resort to numerical methods in order to determine the phases since, in these cases, we will obtain a system of nonlinear equations.

\section{B. RG approach 2: Channel mixing and closing}

The previous RG procedure, although elegant in its simplicity, is by no means the only one which we can apply to our system. If we have two input and two output channels on each face of the large scatterer, then another procedure would be to redistribute the reflected current from that face between the two channels and then close off the appropriate channel on the face.

Consider applying the following transformation to the large scattering matrix in Eq. (8):

$$
\tilde{S}^{\prime}=\tilde{\tau} \tilde{S} \tilde{\tau}^{T}
$$

where $\tilde{\tau}$ is the real block diagonal matrix

$$
\tilde{\tau}=\operatorname{diag}\left(\tilde{R}_{1}, \tilde{R}_{2}, \tilde{R}_{3}\right)
$$

and $\tilde{R}_{j}$ are the usual $2 \times 2$ rotation matrices, $j$ referring to the particular face of the scatterer. This rotation redistributes the current between the channels on each face while maintaining all the symmetries of $\tilde{S}$. Next we close off one channel on each face by feeding the output back into the input channel; see Fig. 4. This can be 

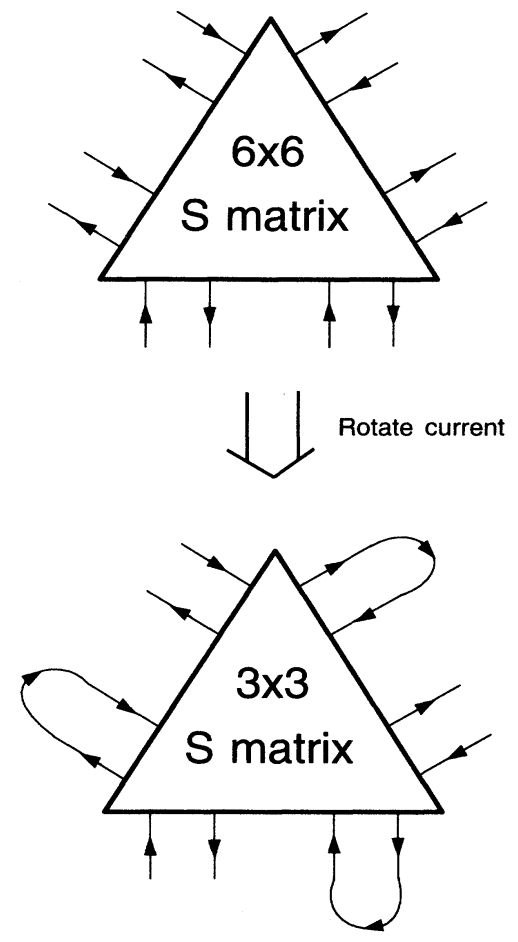

FIG. 4. After performing a rotation on the $6 \times 6$ scattering matrix, the first channel on each face of the large triangular scatterer is closed off to produce the required $3 \times 3$ matrix.

done coherently or incoherently by the introduction of some random phase. Then, rearranging the matrix equation which relates the output amplitudes to the inputs, we obtain an expression for the renormalized scattering matrix which is equivalent to Eq. (7).

The only thing left to determine is the choice of the rotation angles $\phi_{j}$ in the transformation matrix $\tilde{\tau}$. These angles can be chosen in at least two different ways.

\section{Maximize the reflected current into the closed channel}

Let the current reflected back from face $j$ of the large triangular scatterer in Fig. 3(a) be $I_{j}$. If a wave with amplitude $\left(\cos \phi_{j}, \sin \phi_{j}\right)$ enters the scatterer, then the current which is reflected back from face $j$ is

$$
I_{j}=\left(\cos \phi_{j}, \sin \phi_{j}\right) \operatorname{Tr}\left(\tilde{r}_{j} \tilde{r}_{j}^{\dagger}\right)\left(\begin{array}{c}
\cos \phi_{j} \\
\sin \phi_{j}
\end{array}\right),
$$

where $\tilde{r}_{j}$ is the $2 \times 2$ reflection block

$$
\tilde{r}_{j}=\left(\begin{array}{ll}
{ }^{11} r_{j} & { }^{12} r_{j} \\
{ }^{12} r_{j} & { }^{22} r_{j}
\end{array}\right)
$$

What we are really interested in is the transmission through the sample; hence what we aim to do is to redistribute as much of the reflected current as we can into one of the channels and then close it off. In doing so, we hope that the reflected current will decouple from the transmitted current and thus prove unimportant to the transmission. The maximum reflected current occurs when $d I_{j} / d \phi_{j}=0$. Evaluating this derivative leads to the solution

$$
\phi_{j}=\tan ^{-1}\left[\frac{(\gamma-\alpha)}{2 \operatorname{Re}(\beta)} \pm \sqrt{\frac{(\alpha-\gamma)^{2}}{4[\operatorname{Re}(\beta)]^{2}}+1}\right],
$$

where

$$
\begin{aligned}
& \alpha=\left|{ }^{11} r_{j}\right|^{2}+\left|{ }^{12} r_{j}\right|^{2} \\
& \beta={ }^{11} r_{j}^{*}{ }^{12} r_{j}+{ }^{12} r_{j}^{*}{ }^{22} r_{j} \\
& \gamma=\left|{ }^{12} r_{j}\right|^{2}+\left|{ }^{22} r_{j}\right|^{2} .
\end{aligned}
$$

One of the solutions corresponds to the maximum reflected current and the other to the minimum. We choose the phase which minimizes the current reflected into the first channel, which we will leave open. Thus the maximum reflected current has been redistributed into the channel which we will close off. The procedure is then repeated for the other faces of the scattering object.

\section{Choose a random angle}

The simplest possible approach is to choose the rotation angle randomly. In the spirit of a maximum entropy approach, we would then hope to explore all of the rotation angle space. We can do this as long as we make our distribution of scattering objects very large, so that we carry out the RG procedure a sufficient number of times. Unlike the previous method of maximizing the current, this simpler approach does not rely on selecting extremal values.

\section{RESULTS AND DISCUSSION}

We ran a numerical simulation with an initial array of 8192 elements for triangular and square scatterers in $2 \mathrm{D}$ and for cubic scatterers in 3D. Each scatterer constituted an element in our array. The initial array was obtained by randomly generating 8192 scatterers, specified by a disorder parameter. Our method of generating the initial scatterers has been explained elsewhere, ${ }^{8,13}$ but involves combining one-dimensional wires to create the type of scatterer we require. The transmission and reflection of the scatterers can be determined from the transmission and reflection coefficients of the one-dimensional wires, which we select randomly from a disorder dependent distribution. We use the distribution derived by Azbel, ${ }^{26}$

$$
P(|t|)=\left(\frac{1}{W}\right) \frac{1}{|t|}, \quad \exp (-W) \leq|t| \leq 1,
$$

where $W$ is the disorder in the one-dimensional wire and we assume that the reflection and transmission phases are random. With this distribution we are assuming that transmission through the wire is dominated by tunneling via an eigenstate in the wire. The parameter $W$, which 
we term the disorder, should not be confused with the disorder in the Anderson model. ${ }^{1}$ The microscopic picture of the Anderson model requires two parameters to be specified, namely, the energy of the state and the disorder within the system. Ours is a macroscopic model of the transport properties and requires us to specify only one parameter.

The particular RG procedure selected was then performed on the scattering matrices selected from the array to create a new array of scattering matrices on a longer length scale. Once the new array had been created, we discarded the array representing the previous length scale. The procedure was then iterated an arbitrary number of times. Each element of our array could be selected more than once, since they all remained in the array until the new ensemble had been created. To create a new scatterer at every stage would have been too wasteful of CPU time. This must introduce some correlations into the new array of scattering matrices, but it is believed that these will be almost insignificant for an array of 8192 elements. ${ }^{11}$ After the new array had been created, we calculated the conductance of each scattering object using the Landauer formula and studied the behavior of this distribution with system size, initial disorder, and RG procedure.

\section{A. RG approach 1}

We attempted this RG method on triangular scatterers first, because the phase information could be recovered analytically. Unfortunately, though, we found that for many of the large triangular scatterers (around 10\%) that we tried to renormalize, we could not find a real solution to the off-diagonal conditions of Eq. (13) for the transmission phase angles. The problem occurs when only one of the three renormalized transmission amplitudes is small. With no way to find these phases, we had to reject them from the simulation. Although the renormalized scatterers were unphysical in these instances, we know that the conductance is often dominated by rare events and therefore rejecting these scatterers in this way is really not acceptable.

This problem is not restricted to triangular scatterers, unfortunately. We tried to use this RG method for square scatterers. To our disappointment, we were unable to find a numerical method which could reliably determine the required phases as the solutions to a set of six nonlinear equations. The problem was more severe in this case: more than $70 \%$ of the large scatterers we tried to renormalize had to be rejected, because we could not find the root of the equations.

Nevertheless, we present the results of the simulation for triangular scatterers in Fig. 5. Figure 5(a) shows the behavior of $\langle\ln g\rangle$ against the logarithm of the system size, as the RG procedure is iterated. After five or so iterations of the RG procedure, straight lines of gradient $\approx-1$ are achieved for all values of disorder, indicating power law localization. This is rather surprising: Even for strong disorder (a disorder value of 2.0 is strong in our case), we see no evidence of exponential localization as we would expect. Figure 5(b) shows how the variance of $\ln g$
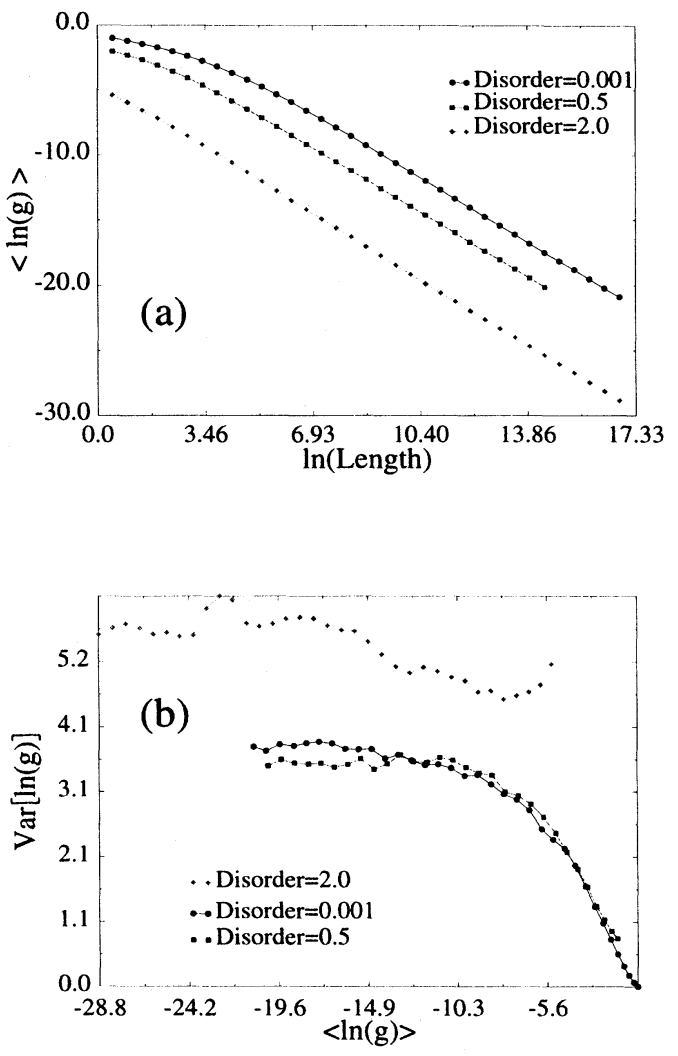

FIG. 5. Graph (a) shows the behavior of $\langle\ln g\rangle$ with system size for the current conservation RG. Note that only power law localization is observed. Graph (b) shows how the variance of the $\ln g$ distribution scales with the mean. The relationship is not linear, as it would be if single parameter scaling was being observed.
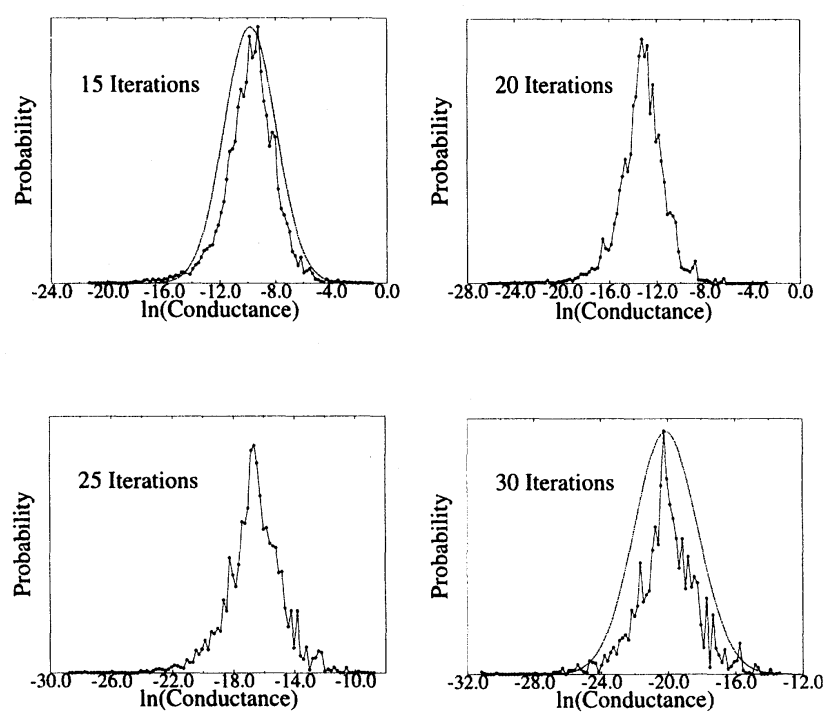

FIG. 6. The behavior of the $\ln g$ distributions is shown for moderate disorder (disorder value 0.5 ). They match less well to the corresponding Gaussians as the RG procedure is iterated. 
scales with $\langle\ln g\rangle$, for various initial values of disorder. The variance is not single valued, indicating that there is no single parameter scaling.

Finally, we show how the conductance distribution evolves with the length of the system (or the number of iterations of the RG procedure) for a moderate disorder value in Fig. 6. Two points are of note when examining these distributions: First, the tails of the distributions are very long, but more importantly the distributions become more spiky and deviate more from the smooth Gaussian form as the RG procedure is iterated. We would expect to see a log-normal distribution for this disorder value, so all these results taken together cast doubt on this RG procedure.

\section{B. RG approach 2}

The channel mixing and closing technique has the advantage that we can always renormalize the scatterers; thus we never lose any elements from our array.

\section{Maximize the reflected current into the closed channel}

In Fig. $7\langle\ln g\rangle$ is plotted against the length of the system, for square scatterers. Encouragingly, we find exponential localization in this case. In fact, no matter how small we made the initial disorder, we found no evidence for anything other than exponential localization. As expected, Table I shows that the localization lengths decreased as the initial disorder of the system was increased.

However, the form of the conductance distributions reveals a major flaw with this RG procedure: The distributions are not log-normal; see Fig. 8. A large peak around the origin is observed and a very long tail towards low conductance quickly develops as the system size increases. The method appears to be too extremal; there is too wide a spread in the conductance measurements on each scatterer in the array.

We repeated the simulation for two-dimensional triangular scatterers and obtained almost identical results.

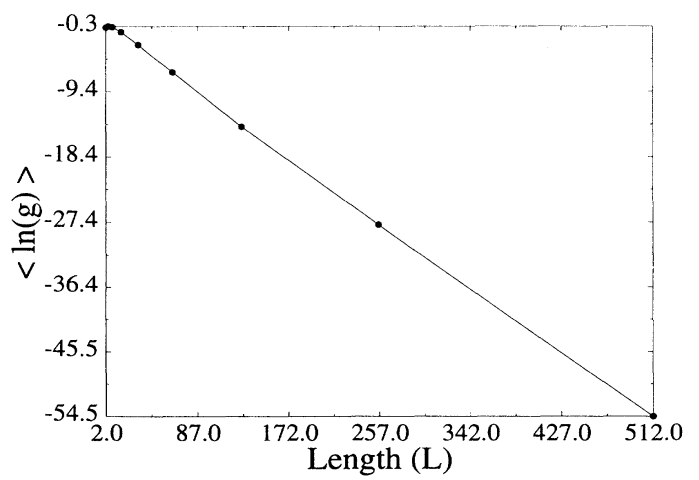

FIG. 7. The behavior of $\langle\ln g\rangle$ with system size is shown for the maximized reflected current RG at moderate disorder. Exponential localization is observed.
TABLE I. Localization length behavior with increasing disorder for the maximized reflected current RG.

\begin{tabular}{cc}
\hline \hline Disorder & Localization length \\
\hline 0.001 & 13.33 \\
0.500 & 9.346 \\
1.000 & 5.987 \\
2.000 & 0.491 \\
\hline
\end{tabular}

\section{Random phase}

Figure 9(a) shows that this RG procedure gives only power law localization. This is true whether we use triangular or square scatterers. In this case the decay is somewhat greater, giving an exponent of $\approx-4$. However, unlike RG approach 1 , we find that single parameter scaling holds for this method. In Fig. 9(b), the variance is plotted against the mean for the $\ln g$ distributions and the points from all the initial disorder values lie on a single straight line, giving the relation

$$
\operatorname{Var}(\ln g) \approx-2\langle\ln g\rangle
$$

for triangular scatterers. If the simulation is repeated for squares, then the gradient changes to $\approx-3$.

In Fig. 10 the $\ln g$ distributions are shown for increasing system size. Superimposed on these numerical distributions, we show the corresponding Gaussian distributions. The agreement between the two is excellent for all system sizes. Unlike the other RG procedures, the smooth Gaussian shape is maintained throughout the iteration sequence. Again, very similar results were obtained on the square scatterers.

\section{Three-dimensional systems}

The random phase RG procedure seemed to work best in 2D. Although no exponential localization could be
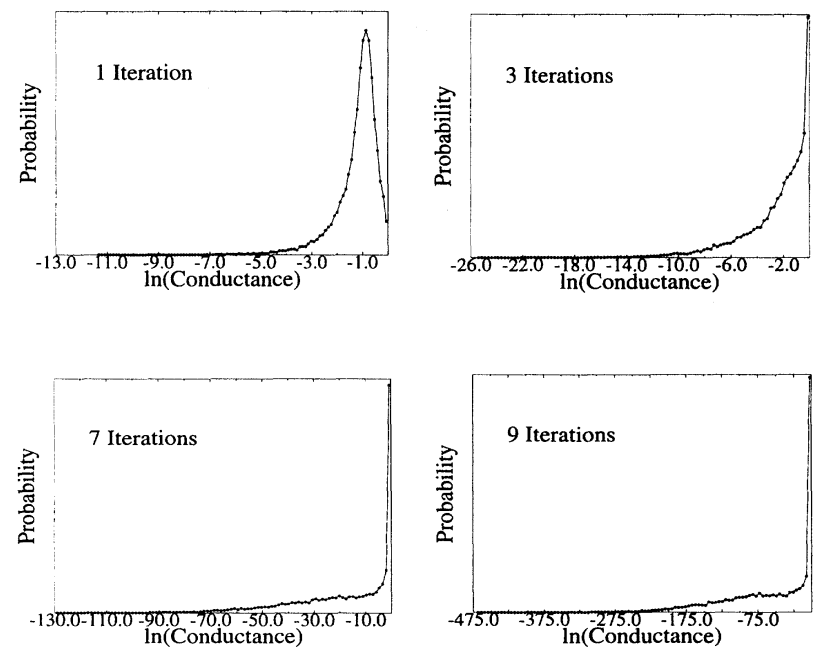

FIG. 8. The behavior of the $\ln g$ distributions is shown for a disorder value of 0.5 . The distributions are not log-normal and develop exceedingly long tails, with a peak near zero. 

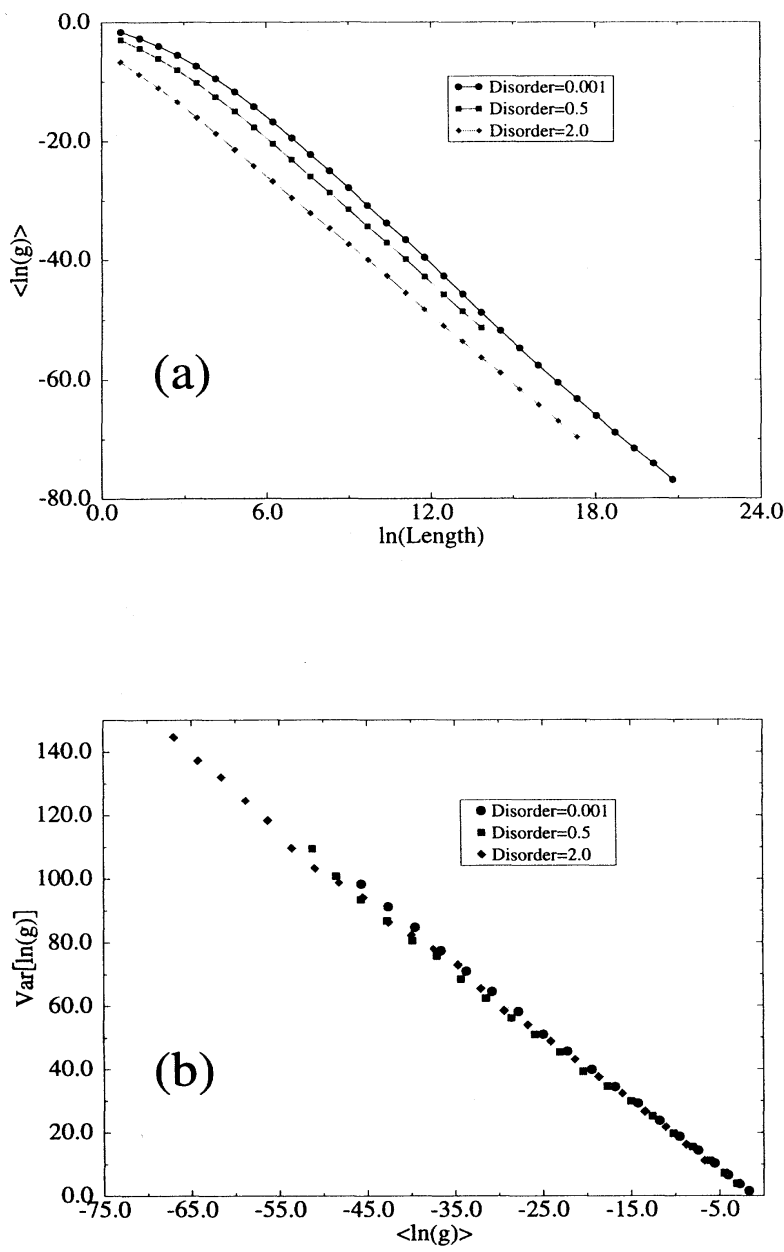

FIG. 9. The behavior of $\langle\ln g\rangle$ with system size shows power law localized behavior in (a). Graph (b) shows a linear relationship between the variance and mean of the $\ln g$ distributions: Single parameter scaling is thus obeyed.

achieved, we decided to employ this technique in $3 \mathrm{D}$. It is, of course, faster and since it is the transition itself which is of interest to us, the fact that the technique did not behave as expected at high disorder may not be a problem in 3D. Unfortunately, however, we could find no evidence of any transition in $3 \mathrm{D}$ with this technique. Just as in $2 \mathrm{D}$, we found only power law localization. But unlike the random phase $R G$ in $2 \mathrm{D}$, we did not find single parameter scaling: The variance of $\ln g$ behaved very much as in Fig. 5(b).

The extension of the maximized reflected current $R G$ to $3 \mathrm{D}$ is not straightforward. Also, given that it did not
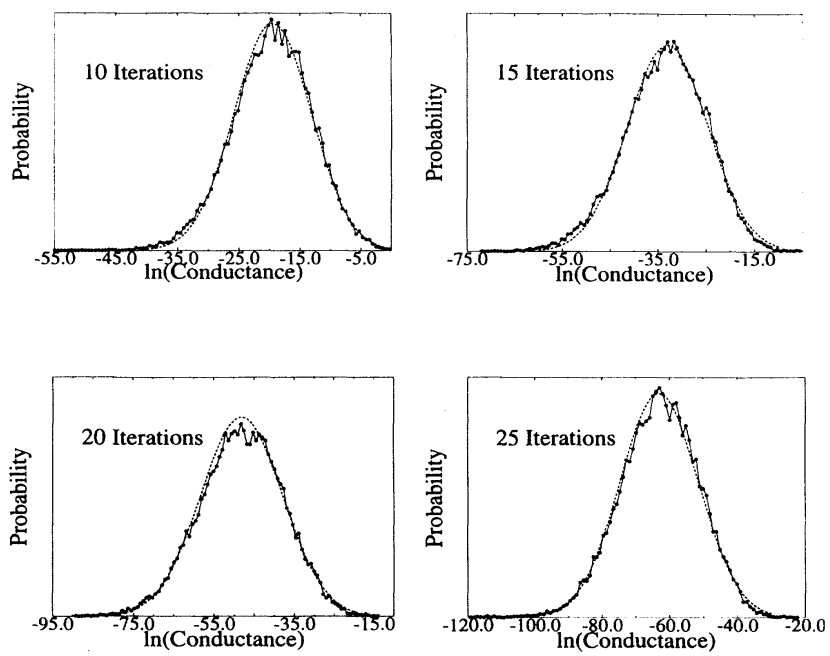

FIG. 10. The behavior of the $\ln g$ distributions with increasing system size; superimposed are the corresponding Gaussians. The numerically obtained distributions are always log-normal.

produce the expected $\ln g$ distributions in $2 \mathrm{D}$, we decided not to test it in $3 \mathrm{D}$.

\section{CONCLUSIONS}

The results of our investigations are summarized for 2D in Table II. Unfortunately, it proved impossible to produce all the expected results with any one of the RG techniques we described in this paper. We expected lognormal conductance distributions in $2 \mathrm{D} . .^{3,4,10,27,28} \mathrm{We}$ also expected that the variance of the $\ln g$ distribution should have scaled with the mean for moderate disorder, but should break down at higher disorder. ${ }^{6,11,29}$ It has proven possible to achieve log-normal distributions of the conductance with variance scaling with the mean, indicating single parameter scaling, with the random phase RG, but no sign of any exponential localization could be achieved with this method. If exponential localization of the conductance is sought, then the minimized reflected current RG must be used. However, in this case the distributions do not resemble anything approaching a lognormal distribution and thus the technique is clearly not realistic. In $1 \mathrm{D}$ the variance is known to vary with the mean as ${ }^{10}$

$$
\operatorname{Var}(\ln g) \approx-2\langle\ln g\rangle
$$

TABLE II. Summary of results of the RG techniques in 2D.

\begin{tabular}{cccc}
\hline \hline & Type of & Log-normal & Single parameter \\
RG technique & localization & distribution & scaling \\
\hline Current conservation & Power law & Probably not & No \\
Maximized reflected current & Exponential & No & No \\
Random Phase & Power law & Yes & Yes \\
\hline \hline
\end{tabular}


in agreement with the random phase RG technique for triangular scatterers. In these simulations, no evidence for the breakdown of single parameter scaling could be found for the random phase RG. However, it can be argued that, since no evidence of exponential localization was detected, single parameter scaling must hold since the system must always be in a regime of low disorder.

These results cast some doubt on the results of Dasgupta et al. ${ }^{23}$ who claim that power law localization does exist for intermediate disorder, in contrast to MacKinnon and Kramer, ${ }^{30}$ who found no evidence of it. Since, for two of our RG methods, we found no other form of localization, it would appear that the very nature of the RG method gives power law localization. Therefore it seems dangerous to claim that power law localization does exist, when other techniques have shown no sign of it and we have shown that RG techniques, when applied to Anderson localization, are not entirely reliable.

Perhaps it is not entirely unsurprising that the RG methods we employed failed in 3D. The very nature of our technique meant replacing all the channels on each face of the scatterer by a single channel. Thus, in 3D the loss of information was greater since the number of channels we had to reduce was greater. Furthermore, the random phase $\mathrm{RG}$ introduces some disorder into the scattering matrix by its very definition. It could be that this small amount of disorder was sufficient to keep the system on the insulating side of the transition.
${ }^{1}$ P. W. Anderson, Phys. Rev. 109, 1492 (1958).

2 E. Abrahams, P. W. Anderson, D. C. Licciardello, and T. V. Ramakrishnan, Phys. Rev. Lett. 42, 673 (1979).

${ }^{3}$ V. I. Melnikov, Fiz. Tverd. Tela (Leningrad) 23, 782 (1981) [Sov. Phys. Solid State 23, 444 (1981)].

4 J. B. Pendry and P. D. Kirkman, J. Phys. C 17, 5707 (1984).

${ }^{5}$ K. M. Slevin and J. B. Pendry, J. Phys. Condens. Matter 2, 2821 (1990)

${ }^{6}$ P. J. Roberts, J. Phys. Condens. Matter 4, 7795 (1992).

${ }^{7}$ E. Hofstetter and M. Schreiber, Europhys. Lett. 21, 933 (1993).

${ }^{8}$ P. M. Bell and A. MacKinnon, J. Phys. Condens. Matter 5, 8337 (1993).

${ }^{9}$ A. MacKinnon, J. Phys. Condens. Matter 6, 2511 (1994).

${ }^{10}$ B. Shapiro, Philos. Mag. B 56, 1031 (1987).

11 A. Cohen, Y. Roth, and B. Shapiro, Phys. Rev. B 38, 12125 (1988).

${ }^{12}$ P. Markoš and B. Kramer, Philos. Mag. B 68, 357 (1993).

${ }^{13}$ P. M. Bell and A. MacKinnon, J. Phys. Condens. Matter 6, 5423 (1994).

${ }^{14}$ A. MacKinnon and B. Kramer, Phys. Rev. Lett. 47, 1546 (1981).
15 J. L. Pichard and G. Sarma, J. Phys. C 14, L127 (1981).

${ }^{16}$ T. J. Godin and Roger Haydock, Phys. Rev. B 38, 5237 (1988).

${ }^{17}$ K. G. Wilson, Phys. Rev. B 4, 3174 (1971).

${ }^{18}$ P. A. Lee, Phys. Rev. Lett. 42, 1492 (1979).

${ }^{19}$ P. A. Lee and D. S. Fisher, Phys. Rev. Lett. 47, 882 (1981).

${ }^{20}$ B. Shapiro, Phys. Rev. Lett. 48, 823 (1982).

${ }^{21}$ S. R. White and R. M. Noack, Phys. Rev. Lett. 68, 3487 (1992).

${ }^{22}$ R. M. Noack and S. R. White, Phys. Rev. B 47, 9243 (1993).

${ }^{23}$ I. Dasgupta, T. Saha, and A. Mookerjee, J. Phys. Condens. Matter 4, 7865 (1992).

${ }^{24}$ R. Landauer, Phys. Scr. T42, 110 (1992).

${ }^{25}$ C. H. W. Barnes, Ph.D. thesis, University of London, 1990.

${ }^{26}$ M. Ya Azbel, Phys. Rev. B 28, 4106 (1983).

${ }^{27}$ P. W. Anderson, D. J. Thouless, E. Abrahams, and D. S. Fisher, Phys. Rev. B 22, 3519 (1980).

${ }^{28}$ P. A. Mello and B. Shapiro, Phys. Rev. B 38, 5860 (1988).

${ }^{29}$ K. M. Slevin and J. B. Pendry, J. Phys. Condens. Matter 2, 2821 (1990).

${ }^{30}$ A. MacKinnon and B. Kramer, Z. Phys. B 53, 1 (1983). 